\title{
Radio Parameters to Enhance Spectrum Utilization in Mixed Environment
}

\author{
Karol Kowalik ${ }^{1}$, Adrian Kliks ${ }^{2}$, Bartosz Musznicki ${ }^{1}$, Michał Kołodziejski ${ }^{1}$, and Paweł Kryszkiewicz ${ }^{2}$ \\ ${ }^{1}$ INEA, Poznań, Poland \\ ${ }^{2}$ Chair of Wireless Communications, Poznan University of Technology, Poznań, Poland
}

https://doi.org/10.26636/jtit.2018.123917

\begin{abstract}
It is believed that $5 \mathrm{G}$ networks will provide 1000 times more capacity than current solutions. One of the keys to achieve that goal is not only the utilization of additional radio bands, but also and foremost, the dynamic and efficient spectrum sharing. To successfully implement it such feature statistical observation and analysis of currently operational legacy systems are required. Comprehensive data on the signal parameters will allow then to determine and tune the approach to simultaneous bandwidth usage by existing and new systems. Therefore, to define and introduce the problem this paper presents a conceptual analysis of IEEE 802.16e based WiMAX network operating in the 3.6-3.8 GHz band on the eve of spectrum sharing introduction.
\end{abstract}

Keywords-5G, bandwidth sharing, spectrum utilization, statistical characteristics, WiMAX.

\section{Introduction}

The 5G wireless networks have to face different rigorous requirements and expectations, broadly defined as 5G Key Performance Indicators (KPIs). Based on [1] and [2], the following KPIs can be identified:

- 1000-fold increase in mobile data volume in certain geographical areas,

- 10 to 100 times higher typical user data rate,

- up to 10 times reduced energy consumption,

- less than $1 \mathrm{~ms}$ end-to-end latency,

- 10 to 100 times increased number of simultaneously connected devices,

- it is assumed that the ubiquitous access will include also low-density areas.

Such a wide set of requirements entails that the delivery of various services to the end-users will be possible by the means of dynamic spectrum aggregation under the umbrella of 5G networks [3]-[6]. Therefore, the on-going improvement of existing radio access methods and new ideas development is required. Prospective enablers include new waveforms [7], massive Multiple Input Multiple Output (MIMO) [8], [9], network visualization, and Software Defined Radio (SDR) [10]-[13]. Some approaches aim to utilize new frequency bands exceeding $6 \mathrm{GHz}$, such as centimeter and millimeter waves [14], [15], as they enable allocation of a wide spectrum for short-range technologies. Alternatively, flexible utilization of sub- $6 \mathrm{GHz}$ frequencies is envisaged as a key enabler for guaranteeing long-distance service delivery and more efficient spectrum utilization.

Given that the spectrum is allocated to different operators, classic coexistence solutions within the licensed and unlicensed portions of the spectrum may not provide the isolation required. Moreover, typical approaches based on orthogonal spectrum sharing between operators within licensed bands may prove inefficient in future scenarios. Hence, a new vision for spectrum utilization is required, and it is highly expected that in the context of future wireless communications systems two traditional models of spectrum management and licensing schemes, i.e. exclusive use and license-exempt, will be complemented by more flexible versions.

One may notice that numerous solutions to advanced resource sharing have been foreseen so far. The examples include infrastructure sharing, as in the Multi-Operator Core Network (MOCN) approach, Licensed Shared Access (LSA), with European standards for the 2.3-2.4 GHz band established by the European Telecommunications Standards Institute (ETSI) [16], or 3-tier sharing model promoted in the USA for the 3.55-3.7 GHz band and known as Citizen Broadband Radio Service with Spectrum Access System (CBRS/SAS) [17]. However, implementation of these standards is still in the trial phase and numerous field-tests and experiments have to be conducted to verify the applicability of the proposed solutions.

At the same time, network operators aim to maximize their revenues from existing infrastructure. One particular example is the utilization of the $3.5 \mathrm{GHz}$ band for new wireless communications systems, as this is the band already allocated to systems such the IEEE 802.16e-2005 [18], based on WiMAX networks. As Long Term Evolution (LTE) and LTE-Advanced (LTE-A) networks develop, the number of customer connected to WiMAX networks declines on 
a continuous basis. So, even though the family of WiMAX standards is not part of $5 \mathrm{G}$ and is considered to be of the legacy variety and nearly extinct, these networks still operate and occupy radio spectrum resources.

However, radio resources associated with the WiMAX technology may be also shared with other technologies. In general, future radio systems may need to share the radio spectrum with legacy communications systems. It is important to analyze the real characteristics describing the existing systems, and to draw conclusions regarding prospective utilization of WiMAX frequency bands for 5G purposes. Therefore, we have performed an initial field analysis concerning key parameters of the WiMAX system which have to be considered while deploying new radio systems. Moreover, considerations have been complemented with relevant observations related to 2.4 and $5 \mathrm{GHz}$ IEEE 802.11 access networks [19]. In that context, the observations and conclusions presented further in this paper may be useful for operators who need to keep their WiMAX networks running in the future, but intend to utilize, in the meantime, the spectrum allocated to them in a more efficient manner, by sharing it with other radio systems.

The remainder of the paper is organized as follows: Section 2 briefly presents deployment of WiMAX within an INEA network and discusses its key system parameters, as well as the spectrum sharing scheme considered. Next two sections present the results of long-term (two years) and short-term (two weeks) WiMAX signal observations. Then, Section 5 introduces general similarities with parameters of 2.4 and $5 \mathrm{GHz}$ IEEE 802.11 networks. Section 6 concludes the paper.

\section{Spectrum Sharing Scenario}

\subsection{INEA's WiMAX Deployment}

INEA is the largest regional fixed-access telecommunications operator in the Greater Poland region and provides various multimedia and connectivity services to over 250,000 of private and business users, relying on different backbone and access technologies, i.e. Gigabit Passive Optical Network (GPON), point-to-point carrier Ethernet optical fibers, IEEE 802.16e WiMAX, IEEE 802.11 Wi-Fi, as well as Hybrid Fiber-Coaxial (HFC), twisted pair-based xDSL and IEEE 802.3 Ethernet [20].

Since 2010, INEA has been rolling-out and maintaining WiMAX-based services aimed to meet the needs of home users scattered across $30,000 \mathrm{~km}^{2}$ within the region [21]. Selection of the IEEE 802.16e-2005 standard and the Time Division Duplexing (TDD) mode made it possible to adjust the downlink/uplink ratio to suit the needs of endusers' data transmissions. Following comprehensive testing of infrastructure and equipment supplied by various vendors, INEA has chosen the Motorola (currently Cambium Networks) PMP320 solution due to its compact form and energy-efficient components. The choice was also in- fluenced by its uncomplicated management and installation, which ensured low cost of ownership. So far, this deployment has provided Internet access and telephony services to almost 6000 households.

\subsection{Key WiMAX Signal Parameters}

As far as WiMAX signal parameters are concerned, particular emphasis should be placed on those which affect system capacity the most. Knowledge of their statistical characteristics may then aid and allow coexistence of two or more systems in the same spectrum, enabling the radio resources available to be shared.

First and foremost, the capacity of a WiMAX network is not fixed [22]. Each Customer-Premises Equipment (CPE) operates with spectral efficiency that changes in time and is influenced by three parameters: modulation, Forward Error Correction (FEC) coding and Multiple Input Multiple Output (MIMO) mode. Therefore, the network capacity of a given access point (AP) is a function of the number of CPEs and their spectral efficiency. For example, if one CPE operates with the modulation of 64-QAM, then its spectral efficiency is $6 \mathrm{bits} / \mathrm{s} / \mathrm{Hz}$. If it is using $5 / 6 \mathrm{FEC}$, then the spectral efficiency is reduced to $5 \mathrm{bits} / \mathrm{s} / \mathrm{Hz}$, whereas for MIMO-B its spectral efficiency is doubled and reaches about 10 bits/s/Hz. The three parameters: modulation, FEC coding and MIMO mode are mostly affected by Received Signal Strength Indication (RSSI) and Carrier to Interference and Noise Ratio (CINR) values. Therefore, the following analyses are focused on RSSI and CINR statistical changes.

\subsection{Spectrum Sharing of $3.5 \mathrm{GHz}$ Radio Resources}

As it has been pointed out, it is usually economically justified for the network operator to maximize their revenues from the existing infrastructure and from the spectrum licenses held. If it was possible to either offer new services by relying on the existing infrastructure or utilize the available spectrum resources in a more efficient way, such an approach would be highly beneficial for the network operator.

In INEA's case, the second option is being considered, as the WiMAX network has to be kept operational (as the highest priority, incumbent network). Therefore, the following question arises: can other parallel services be deployed, in particular microwave lines or other point-to-point transmissions, that will seamlessly operate in the $3.5 \mathrm{GHz}$ band?

To make such a solution possible, stable methods for the protection of incumbent (WiMAX) network from harmful interference have to be implemented. However, this will be only possible if the key operating parameters that describe the behavior of the WiMAX network are precisely identified. For example, in order to calculate the impact of the induced interference originating from the new network on 
the WiMAX network, one needs to know what the required average values observed in the downlink (DL) and uplink (UL) transmission directions in the WiMAX system are. It is also possible however, to analyze the potential changes of these parameters over time. Thus, keeping in mind the main objective of this research project, i.e. is future coexistence of two wireless systems operating in the same frequency band, the present study focuses on the analysis of the changes in RSSI and CINR values measured, as a function of time. Two approaches have been tested, i.e. 2-year long observations to detect long-term trends, further discussed in the context of short, 2-week long measurement campaigns.

\section{Long-term Signal Observations}

At INEA, every CPE is queried once every hour with the use of the Network Diagnostics System (NDS) to collect statistical data about the quality of the connection. These queries are typically performed using the Simple Network Management Protocol (SNMP), and the results are stored for at least one year. In the case of the WiMAX network, data for last two years has been obtained, covering the period from April 2015 to April 2017. This data is based on information collected from about $6000 \mathrm{CPEs}$ and includes various network parameters (such as MAC address, IP address, base station ID), and two PHY layer signal parameters:

- RSSI for both downlink and uplink,

- CINR for both downlink and uplink.

First of all, the distribution of RSSI and CINR values should be analyzed. Figure 1 presents the distribution of average RSSI values in downlink and uplink directions.

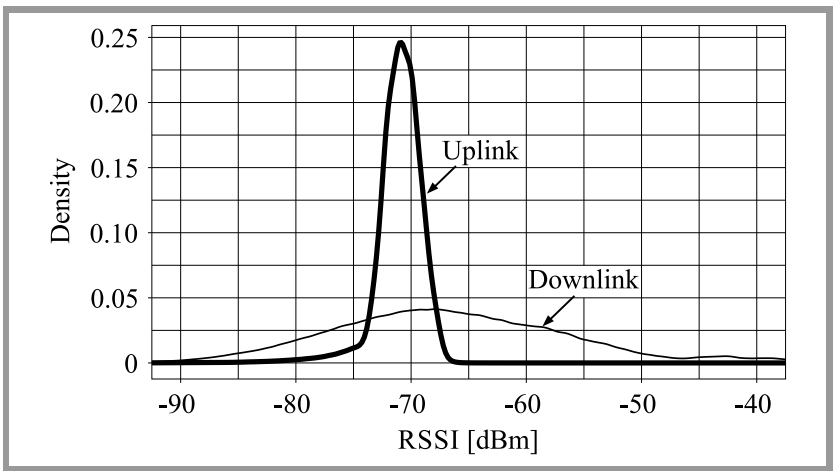

Fig. 1. Distribution of RSSI.

It can be observed that DL RSSI values are spread across a wide range of values, from -50 to $-90 \mathrm{dBm}$, with the mean value at $-66 \mathrm{dBm}$, while UL RSSI values oscillate closely around the mean at $-71 \mathrm{dBm}$. This can be explained by the transmit power control mechanism implemented for uplink, as the WiMAX access point imple- ments a power control mechanism to mitigate path loss, shadowing, etc.

The access point is then configured with a target RSSI value, which is set, in INEA's network, at $-71 \mathrm{dBm}$. Therefore, WiMAX access points instruct all the connected CPEs to adjust their transmit (TX) power to meet the target RSSI value. Hence, the RSSI values are distributed closely to the mean value which, in turn, is equal to the configured target RSSI value. In the downlink direction, each access point transmits at maximum power, and, therefore, all DL RSSI values are proportional to the path loss, shadowing, etc. Thus, the range of values for DL RSSI is much wider than for UL RSSI.

As it is visible in Fig. 2, the CINR density function follows trends that are similar to RSSI, and DL values occupy a much wider range than UL. It can be observed that DL CINR values are ranging from 15 to $40 \mathrm{~dB}$, with the mean at $30 \mathrm{~dB}$, while the most of UL CINR values are located within the range of 25 to $33 \mathrm{~dB}$, with the mean at $28.5 \mathrm{~dB}$.

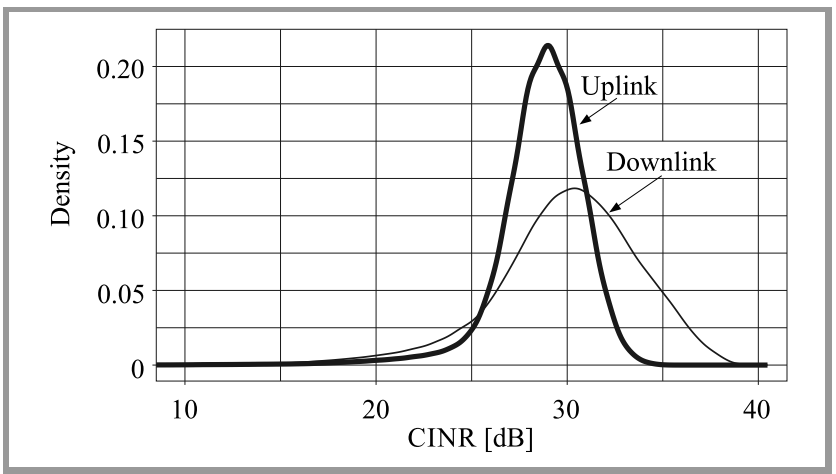

Fig. 2. Distribution of CINR.

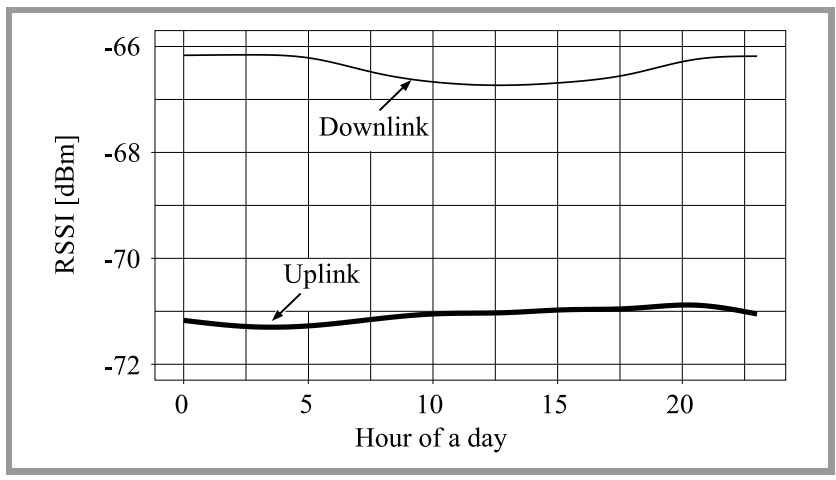

Fig. 3. Average RSSI.

Moreover, signal parameter variations during an average day have been examined. They are calculated as an average for all of CPEs during the two-year observation period. Figures 3 and 4 depict the average RSSI and CINR values as observed round-the-clock. It can be noticed that the values oscillate around the mean values, and that the variation is much smaller for UL parameters than for DL. Moreover, one can observe that DL RSSI values are inversely proportional to the expected seasonal temperature trends. 


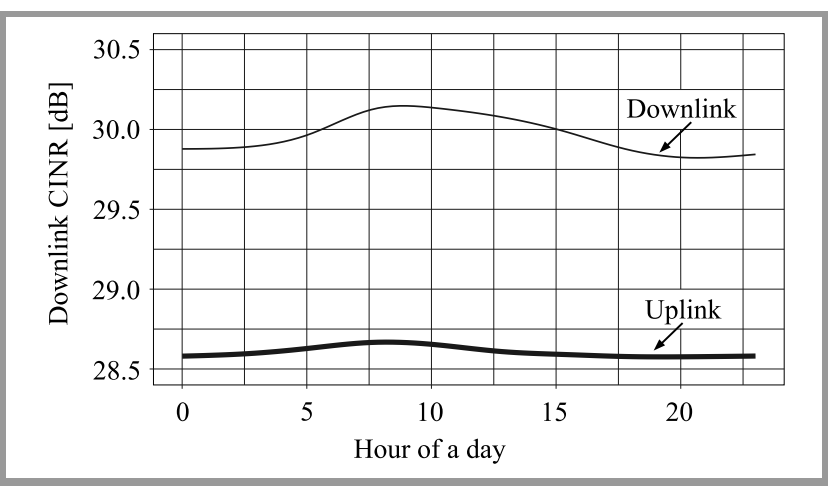

Fig. 4. Average CINR.

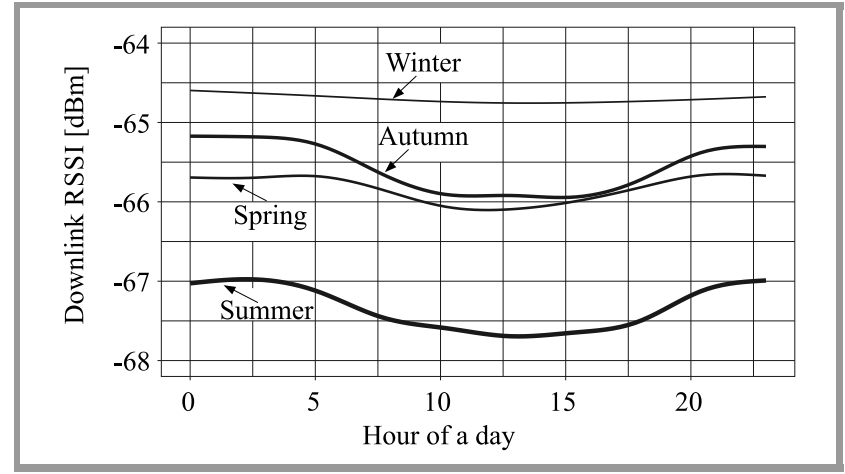

Fig. 5. Average RSSI for each season.

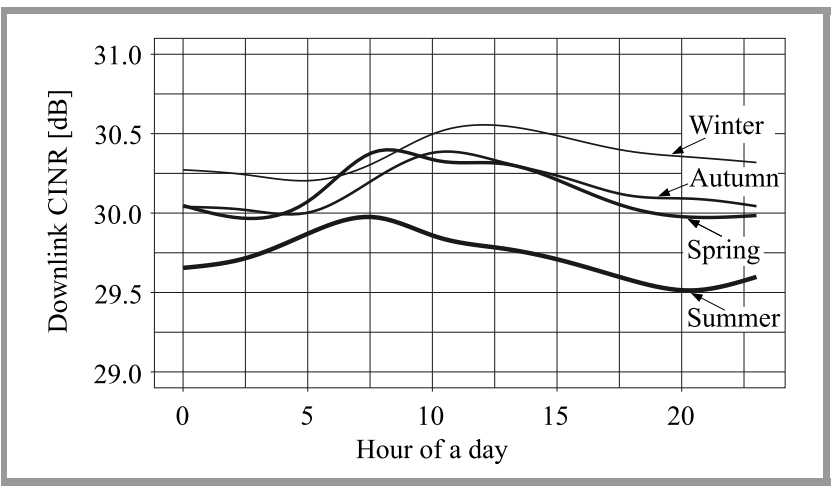

Fig. 6. Average CINR for each season.

To verify if there is any correlation between the RSSI values and temperature, downlink RSSI and CINR for different seasons of the year (calculated as an average for all CPEs during a given season or the two-year observation period) have been plotted in Figs. 5 and 6 respectively. Indeed, Fig. 5 suggests that there is an inverse correlation between RSSI and temperature, because the highest RSSI values can be observed during winter, while in spring and autumn RSSI values are lower and also exhibit a significant decrease during daylight hours. Accordingly, in summer, RSSI values are the lowest. Figure 6 shows some ambivalent trends though. Apparently, in each season a mid-day CINR increase is observed. Still, in summer the CINR values are the lowest, while their highest levels are recorded in winter.

\section{Short-term Signal Observations}

Since two years of observations presented in the previous section did not exhibit a clear correlation between CINR values and temperature, we have decided to capture more dense data from the WiMAX network, and additionally combine it with humidity and temperature values from the Industrial Institute of Agricultural Engineering (PIMR)

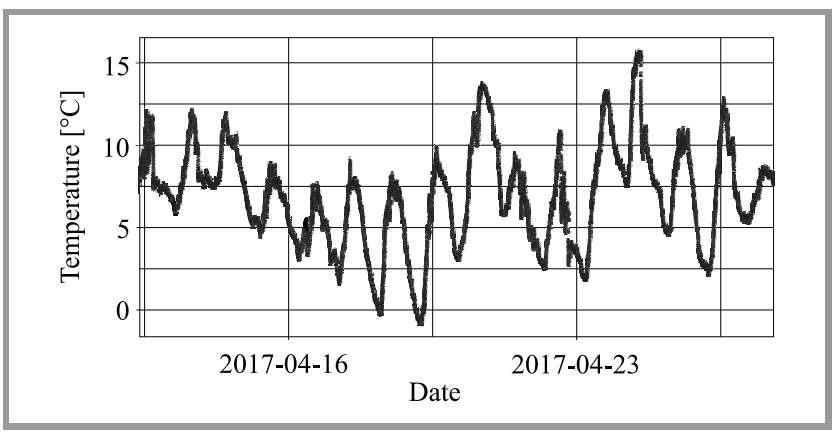

Fig. 7. Temperature for the two weeks period.

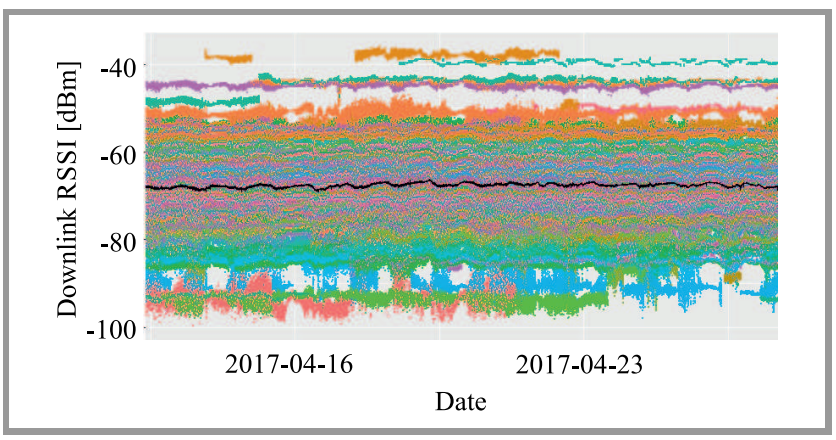

Fig. 8. Download RSSI for the two weeks period. (See color pictures online at www.nit.eu/publications/journal-jtit)

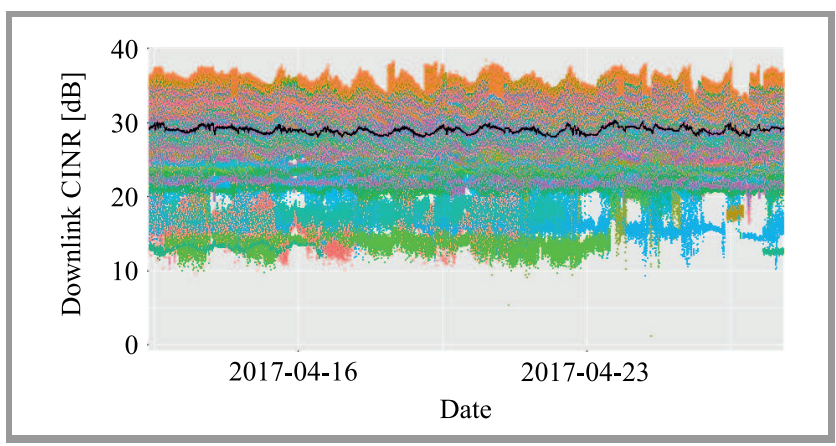

Fig. 9. Download CINR for the two weeks period.

weather station located in Poznan, Poland ${ }^{1}$. In this experiment, 200 WiMAX CPEs located in close proximity to the PIMR weather station have been used. Each CPE has been queried using SNMP every minute during a two-week period between 14 and 28 April 2017. In this way, the following parameters have been collected:

\footnotetext{
${ }^{1}$ Data are available at http://www.pimr.poznan.pl/bup/gethd2003.php
} 
- RSSI for both downlink and uplink,

- CINR for both downlink and uplink,

- average throughput (expressed in $\mathrm{kbit} / \mathrm{s}$ ) for both downlink and uplink,

- Modulation and Coding Scheme (MCS) index for both DL and UL,

- TX power for UL,

- temperature,

- humidity.

As a reference, Fig. 7 demonstrates varying temperature values during the two-week period of interest.

Due to frequent and transient changes of RSSI and CINR, RSSI and CINR mean values for all 200 CPEs have been calculated. These mean trend lines, as well as data points for each minute within the two weeks for all CPEs, are shown in Fig. 8 and 9, respectively. Each of the 200 CPEs was assigned with a different color and the black line in the middle represents the mean value for all CPEs. The mean trajectory expresses a trend which is not specific to any particular CPE, but to the whole network under consideration.

\subsection{Correlation between Parameters}

To analyze the correlation between CINR, RSSI and other network and environmental parameters, a correlation matrix has been plotted, as shown in Fig. 10. This matrix presents the Pearson correlation between all pairs of parameters.

An in-depth analysis of the parameter correlation matrix offers different interesting insights, such as:

- mean DL CINR exhibits positive correlation with temperature and negative correlation with humidity and mean DL RSSI,

- mean DL RSSI exhibits negative correlation with temperature and mean DL CINR,

- mean DL RSSI seams to affect DL CINR and DL MCS index,

- mean DL CINR also seems to affect DL MCS index,

- DL throughput is correlated with UL throughput (since Transmission Control Protocol (TCP) based data transfer required communication in both directions),

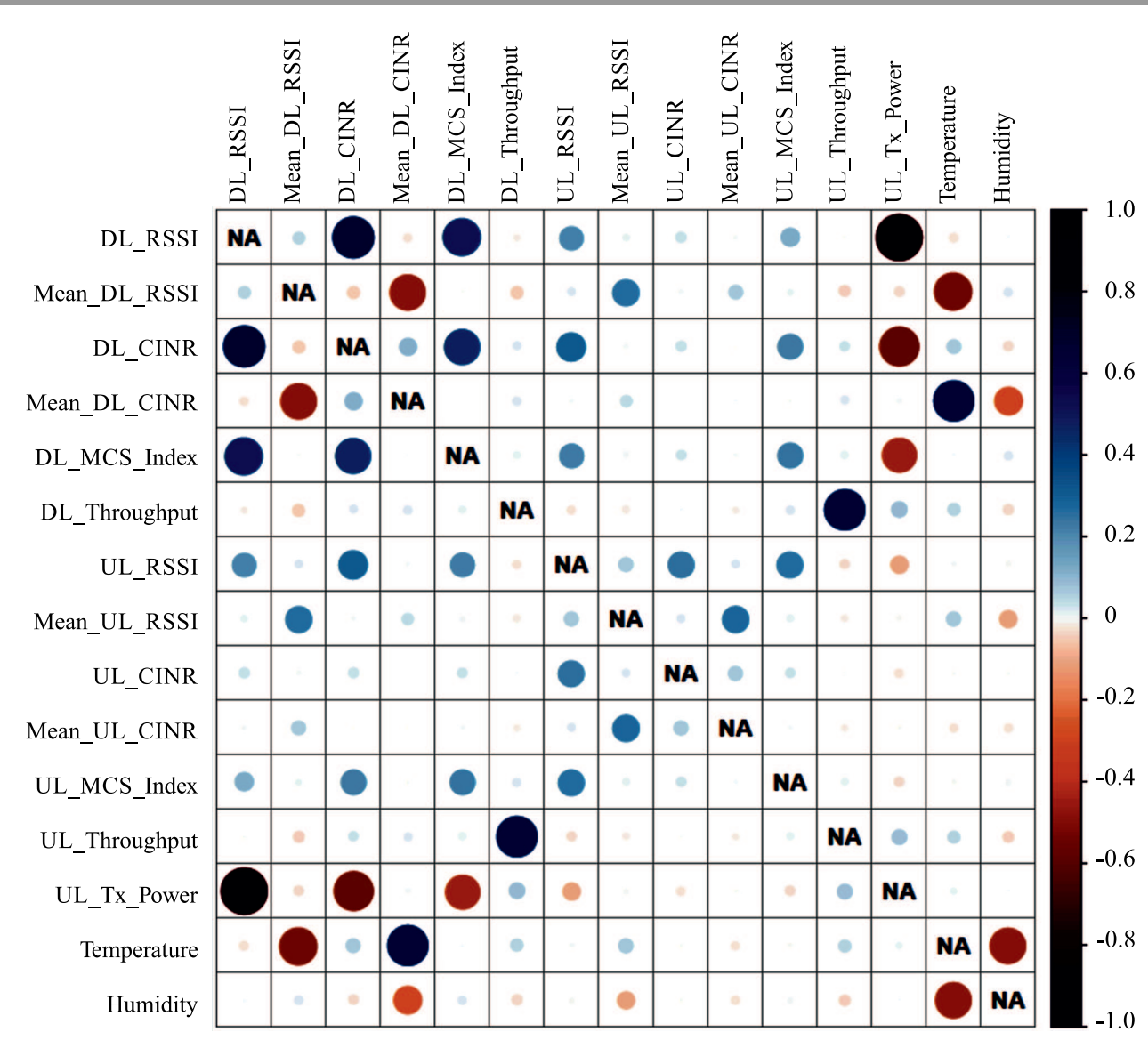

Fig. 10. Parameters correlation matrix. 
- most of uplink parameters do not show any strong correlation with any other parameter, except of uplink TX power which affects DL RSSI, DL CINR and DL MCS index. We consider this finding as the most intriguing, since we cannot identify any reason for such a behavior. TX power on the UL is controlled by adaptive power control, and it should mitigate path loss and shadowing on the UL without affecting DL parameters.

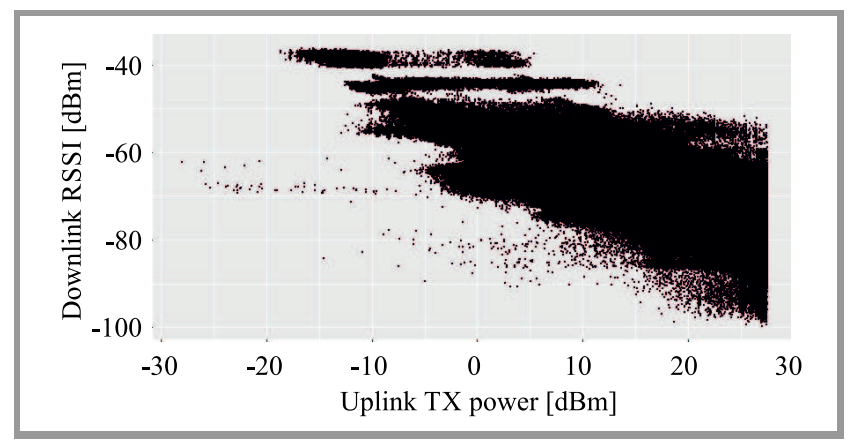

Fig. 11. UL TX power vs. DL RSSI.

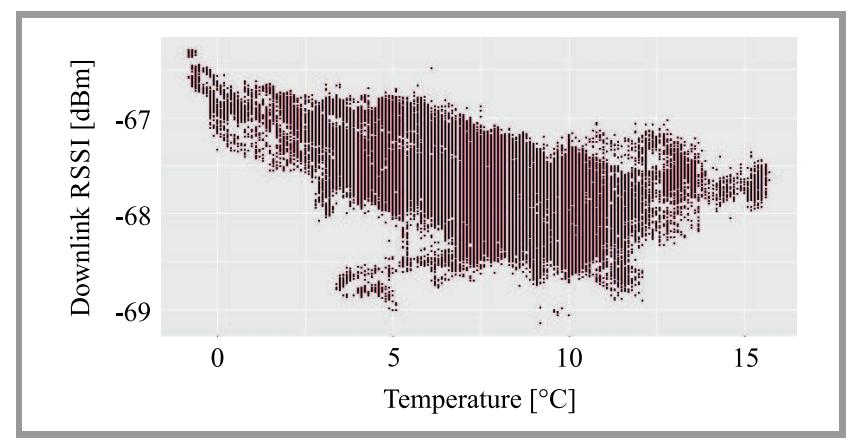

Fig. 12. Temperature vs. DL RSSI.

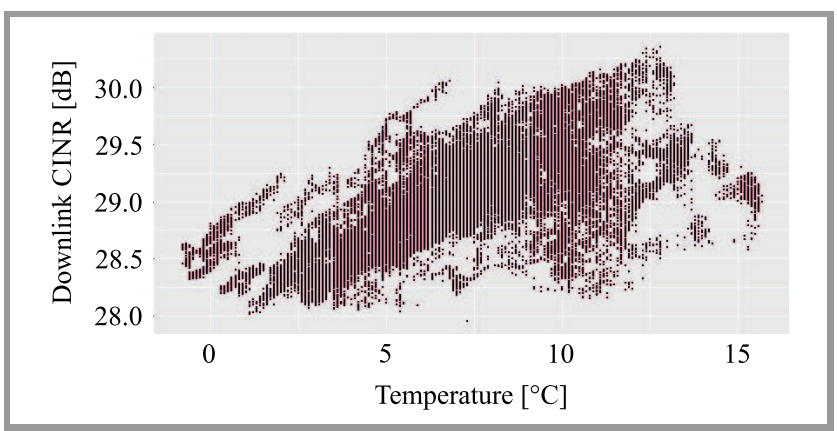

Fig. 13. Temperature vs. DL CINR.

To investigate the correlation between parameters further, we have plotted three pairs exhibiting the highest and almost linear correlation with some limited variations. In Fig. 11 negative correlation between DL RSSI and UL TX power is visible. Figure 12 presents negative DL RSSI correlation with temperature. Conversely, in Fig. 13 positive DL CINR correlation with the temperature can be observed.

\section{Neighboring Radio Bands}

In order to perceive changes in WiMAX signal characteristics in a broader sense, it is worthwhile to refer to similar parameters of widely utilized neighboring Internetbearing unlicensed radio bands. As a matter of fact, the present research has been partially inspired by an earlier study conducted at INEA between 10 June and 10 July 2016 [23], where 330 mobile $2.4 \mathrm{GHz}$ and 10 access points were used. Mobile access points were mounted on-board public buses and trams in Poznań and Konin. Their main everyday task is to provide passengers (and other nearby users) with a Wi-Fi service. Each device is a RouterBoard RB751U equipped with a $2.5 \mathrm{dBi}$ omnidirectional antenna and a $4 \mathrm{G}$ network modem to provide Internet connection. Each of the selected stationary access points is based on Router Board RB433 connected to a 16 or $19 \mathrm{dBi}$ external sector antennas.

Data has been obtained using SNMP queries issued every 15 minutes to collect information on the noise floor level perceived by each AP, and the RSSI value for each connected client. The sampling interval was chosen to guarantee the frequency required to ensure a complete picture of round-the clock variations, without affecting operation of the access point and the end-user experience.

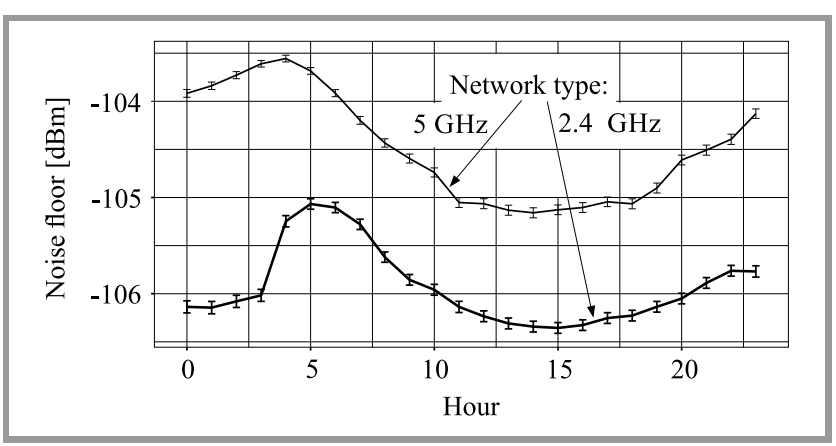

Fig. 14. Average noise floor in 2.4 and $5 \mathrm{GHz}$ networks.

As a result, RSSI for $5 \mathrm{GHz}$ network and noise floor characteristics presented in Fig. 14 have been obtained. It is clearly visible that their shapes resemble the curvature of average RSSI in a $3.5 \mathrm{GHz}$ WiMAX network in summer, as depicted in Fig. 5. Most interestingly, 2.4 and $5 \mathrm{GHz}$ networks operated in seemingly different environmental con-

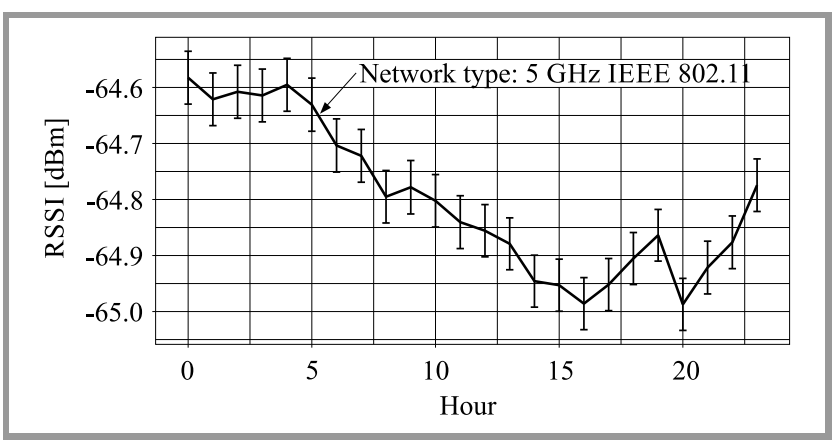

Fig. 15. Average RSSI in a $5 \mathrm{GHz}$ network. 


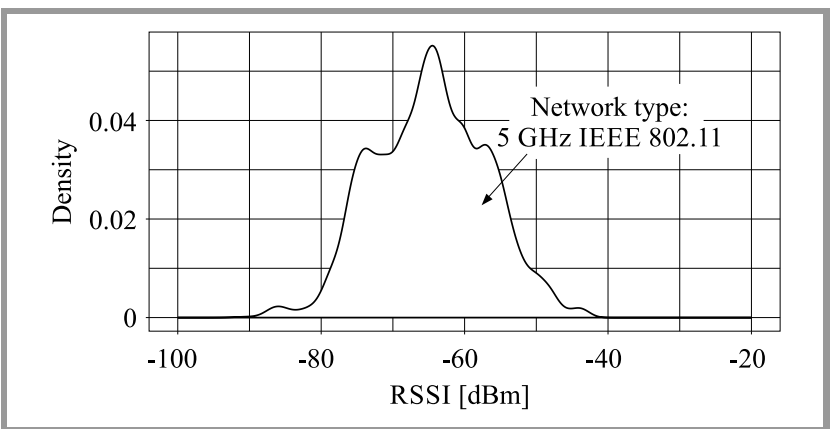

Fig. 16. Distribution of RSSI in a $5 \mathrm{GHz}$ network.

ditions, and yet, the similarities to WiMAX are striking. Access points were not only operating in separated radio bands, but were also equipped with antennas of significantly different gains, and thus, exposed to different angles of noise sources. Mobile APs were traversing busy metropolitan streets, while stationary access points were mounted on aerial masts in more scattered locations. Due to their fixed position, it is also beneficial to refer to the comparable shape of average RSSI in the $5 \mathrm{GHz}$ network presented in Fig. 15. The similarities with WiMAX parameters can be further illustrated by $5 \mathrm{GHz}$ RSSI distribution, as depicted in Fig. 16.

\section{Conclusions}

In this paper, we have presented statistical characteristics of actual, IEEE 802.11e compliant WiMAX signals, as seen from the perspective of improving spectrum utilization by means of simultaneous use of given frequency bands by two wireless systems. As interference will play a key role in such a scenario, in our discussion we have revealed the changes of RSSI and CINR parameters as functions of time. Based on the highlighted results, the following conclusion can be drawn.

There is a direct correlation between, firstly, the mean RSSI and CINR values observed, and, secondly, the season and time of the day. However, in the context of season changes, these variations are rather negligible (around $1 \mathrm{~dB}$ ), and in practice, an appropriate interference margin can be included to reflect these changes.

On the other hand, there are high variations of RSSI and CINR changes observed, noticeable in a shorter time scale. When analyzed per CPE, not per network, as the average RSSI and CINR, they are again more or less stable. These may reach even up to a few $\mathrm{dB}$, and such a change has to be taken into consideration while deploying a new and parallel wireless network. Finally, the specificity of the WiMAX system (i.e. power steering in upload direction) entails the need for the system to adjust to the changes in ambient environment in UL, probably without any loss in average RSSI or CINR. The key problem will affect DL, and due for that circumstance, application of careful network planning algorithms may be needed. The analysis presented has demonstrated that some changes in transmission pa- rameters are natural even in the fixed (stationary) WiMAX network that INEA operates. It is also worth pointing out that spectrum sharing requires long-term measurements of the parameters of the entire radio system (WiMAX in the presented case) in order to obtain key characteristics of the signal and to identify bottlenecks. For example, deterioration in CINR values must not necessarily be due to interference from the secondary spectrum user.

It may be due to ambient temperature (or other external factors) as shown in the article, both for $3.5 \mathrm{GHz}$ WiMAX and for 2.4 and $5 \mathrm{GHz}$ networks. In order to explain it with a higher level of certainty, additional investigation of a broader scope and nature seems to be required. Moreover, the presented study suggests that various not obvious relations between observed parameters may emerge, which indicates that further research is required in order to explain the causes of and the practical significance of the findings. As it has been stressed, even though the WiMAX technology is currently considered outdated, networks based on this technology still operate and occupy radio spectrum resources. Moreover, they often have to be maintained due to the legal commitments towards customers, and this will still be the case, at least in the nearest future. Therefore, the findings presented may be of key importance for operators who need to continue maintaining WiMAX networks and use the spectrum more efficiently in the presence of other (new) systems coexisting in the same band. Further studies and results of similar nature may be also valuable to equipment vendors and regulatory offices to aid their efforts aiming to optimize utilization of the spectrum.

\section{Acknowledgement}

The work has been funded by the EU H2020 project COHERENT (contract no. 671639).

\section{References}

[1] "What Will The 5G-Infrastructure-PPP Deliver?", 5G PPP: The 5G Infrastructure Public Private Partnership [Online]. Available: https://5g-ppp.eu/kpis/

[2] " $5 \mathrm{G}$ Vision - The 5G Infrastructure Public Private Partnership: the next generation of communication networks and services", 5G PPP: The 5G Infrastructure Public Private Partnership, 2015 [Online]. Available: https://5g-ppp.eu/wp-content/uploads/2015/02/5GVision-Brochure-v1.pdf

[3] M. Mustonen, M. Matinmikko, M. Palola, S. Yrjölä, and K. Horneman, "An evolution toward cognitive cellular systems: licensed shared access for network optimization", IEEE Commun. Mag., vol. 53 , no. 5 , pp. $68-74,2015$.

[4] A. Kliks, O. Holland, A. Basaure, and M. Matinmikko, "Spectrum and license flexibility for 5G networks", IEEE Commun. Mag., vol. 53, no. 7, pp. 42-49, 2015.

[5] H. Bogucka, P. Kryszkiewicz, and A. Kliks, "Dynamic spectrum aggregation for future 5G communications", IEEE Commun. Mag., vol. 53 , no. 5 , pp. $35-43,2015$.

[6] R. H. Tehrani, S. Vahid, D. Triantafyllopoulou, H. Lee, and K. Moessner, "Licensed spectrum sharing schemes for mobile operators: A survey and outlook", IEEE Commun. Surv. and Tutorials, vol. 18, no. 4, pp. 2591-2623, 2016. 
[7] P. Banelli, S. Buzzi, G. Colavolpe, A. Modenini, F. Rusek, and A. Ugolini, "Modulation formats and waveforms for $5 \mathrm{G}$ networks: Who will be the heir of OFDM?: An overview of alternative modulation schemes for improved spectral efficiency", IEEE Signal Process. Mag., vol. 31, no. 6, pp. 80-93, 2014.

[8] M. Agiwal, A. Roy, and N. Saxena, "Next generation 5G wireless networks: A comprehensive survey", IEEE Commun. Surv. and Tutorials, vol. 18, no. 3, pp. 1617-1655, 2016.

[9] T. L. Marzetta, "Noncooperative cellular wireless with unlimited numbers of base station antennas", IEEE Trans. Wireless Commun., vol. 9, no. 11, pp. 3590-3600, 2010.

[10] C. Liang and F. R. Yu, "Wireless Network Virtualization: A Survey, Some Research Issues and Challenges", IEEE Commun. Surv. and Tutorials, vol. 17, no. 1, pp. 358-380, 2015.

[11] I. Khan, F. Belqasmi, R. H. Glitho, N. Crespi, M. Morrow, and P. Polakos, "Wireless sensor network virtualization: A survey", IEEE Commun. Surv. and Tutorials, vol. 18, no. 1, pp. 553-576, 2015 (doi: 10.1109/COMST.2015.2412971).

[12] A. Blenk, A. Basta, M. Reisslein, and W. Kellerer, "Survey on network virtualization hypervisors for software defined networking", IEEE Commun. Surv. and Tutorials, vol. 18, no. 1, pp. 655-685, 2015 (doi: 10.1109/COMST.2015.2489183).

[13] Y. Li and M. Chen, "Software-defined network function virtualization: A survey", IEEE Access, vol. 3, pp. 2542-2553, 2015.

[14] T. S. Rappaport, R. W. Heath, R. C. Daniels, and J. N. Murdock, Millimeter Wave Wireless Communications. Prentice Hall, 2015.

[15] S. Kutty and D. Sen, "Beamforming for millimeter wave communications: An inclusive survey", IEEE Commun. Surv. and Tutorials, vol. 18, no. 2, pp. 949-973, 2016.

[16] ETSI TS 103154 V1.1.1. - "Reconfigurable Radio Systems (RRS); System requirements for operation of Mobile Broadband Systems in the $2300 \mathrm{MHz}-2400 \mathrm{MHz}$ band under Licensed Shared Access (LSA)" [Online]. Available: http://www.etsi.org/deliver/etsi_ts/ 103100_103199/103154/01.01.01_60/ts_103154v010101p.pdf

[17] Federal Communications Commission FCC 15-55, Order on reconsideration and second report and order in the matter of amendment of the commission's rules with regard to commercial operations in the 3550-3650 MHz band, May 2016 [Online]. Available: http://transition.fcc.gov/Daily_Releases/Daily_Business/2016/ db0502/FCC-16-55A1.pdf

[18] IEEE Std 802.16e-2005 - IEEE Standard for Local and Metropolitan Area Networks - Part 16: Air Interface for Fixed and Mobile Broadband Wireless Access Systems - Amendment for Physical and Medium Access Control Layers for Combined Fixed and Mobile Operation in Licensed Bands, 2006.

[19] IEEE Std 802.11-2012 - IEEE Standard for Information technology, Part 11: Wireless LAN Medium Access Control (MAC) and Physical Layer (PHY) Specifications, 29 March 2012 [Online]. Available: http://ieeexplore.ieee.org/stamp/stamp.jsp?tp=\&arnumber=6178212

[20] P. Walkowiak, R. Szalski, B. Musznicki, D. Dudek, K. Kowalik, and P. Zwierzykowski, "Evaluation of CARMNET System in INEA HOTSPOT Network", in Proc. of IEICE Inform. and Commun. Technol. Forum IEICE ICTF, Poznań, Poland, 2014 [Online]. Available: http://i-scover.ieice.org/proceedings/ICTF/2014/pdf/ALIEN-4.pdf

[21] K. Kowalik, D. Dudek, M. Kołodziejski, B. Musznicki, E. Grzybek, and J. Jarzina, "Lessons Learned from WiMAX Deployment at INEA", J. of Telecommun. and Inform. Technol., no. 3, pp. 34-41, 2014. [Online]. Available: http://www.itl.waw.pl/czasopisma/JTIT/ 2014/3/34.pdf

[22] C. So-In, R. Jain, and A.-K. Tamimi, "Capacity evaluation for IEEE 802.16e mobile WiMAX", J. of Comp. Syst., Netw., and Commun., Special issue on WiMAX, LTE, and WiFi Interworking, vol. 2010, Article ID 279807, 2010 (doi: 10.1155/2010/279807).

[23] B. Musznicki, K. Kowalik, P. Kłodziejski, and E. Grzybek, "Mobile and residential INEA Wi-Fi hotspot network", in Proc. 13th Int. Symp. on Wireless Commun. Syst. ISWCS 2016, Poznań, Poland, 2016.

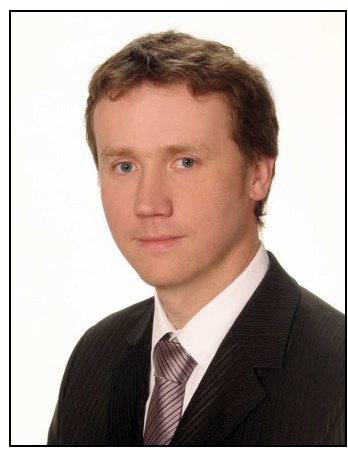

Karol Kowalik received his M.Sc. from Poznan University of Technology in 2000 and Ph.D. from Dublin City University in 2004. He is currently the Technical Development Manager at INEA and is responsible for technical innovation and validation on new ideas. His research interests include networking, switching, routing.

E-mail: karol.kowalik@inea.com.pl

INEA S.A.

Wysogotowo, Wierzbowa st 84

62-081 Przeźmierowo

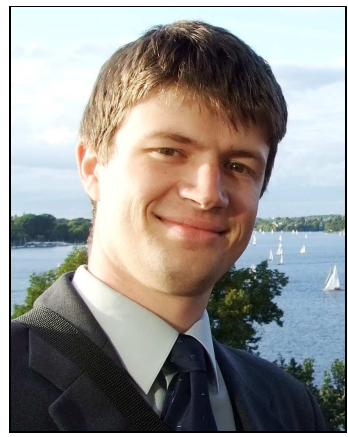

Adrian Kliks received his M.Sc. and Ph.D. degrees in telecommunication (with a distinction) from the Poznan University of Technology, in 2005 and 2011, respectively. Since 2011, he has been working at the chair of wireless communications, holding the position of an Assistant Professor. His research interests cover a wide spectrum of wireless communications. Kliks is particularly interested in new waveforms for future wireless systems, including (non-)orthogonal and noncontiguous multicarrier schemes, in application of cognitive radio technology, in advanced spectrum management, but also in deployment and resource management in small-cells, and network virtualization. He is the author of approximately 100 scientific publications or presentations for technical conferences and journals.

E-mail: adrian.kliks@put.poznan.pl Chair of Wireless Communications

Faculty of Electronics and Telecommunications

Poznan University of Technology

M. Skłodowskiej-Curie sq. 5

60-965 Poznań, Poland

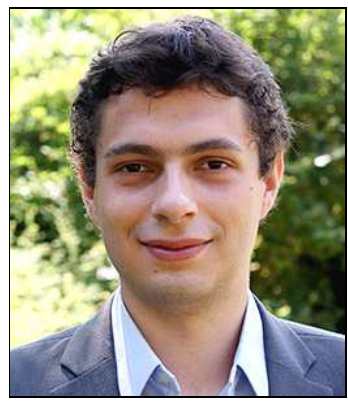

Bartosz Musznicki is pursuing Ph.D. at Poznan University of Technology. His main research interests include topology control and routing in wireless networks. He gained network engineering and managerial experience at INEA. $\mathrm{He}$ is an author of four book chapters, seven journal articles,

and six conference papers. 
E-mail: bartosz.musznicki@inea.com.pl

INEA S.A.

Wysogotowo, Wierzbowa st 84

62-081 Przeźmierowo

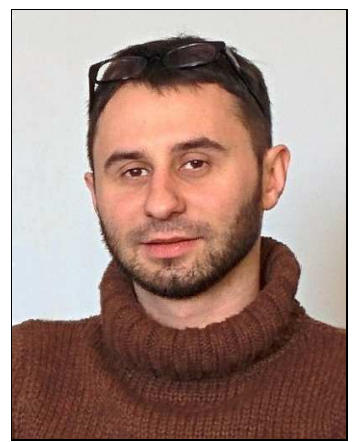

Michał Kołodziejski received M.Sc. in Electronics and Telecommunications from Szczecin University of Technology in 2008. In the meantime, he spent six months at Technical Faculty CAU Kiel in Germany. After graduation, he worked at Atos IT Services Poland in Bydgoszcz as a Network Operator (WAN, LAN monitoring and management). In 2010 he joined INEA in Poznań. Currently he is a radio communications engineer and senior network administrator. He deals with various technologies, especially Wi-Fi, WiMAX, microwave links and customer distribution cable networks.

E-mail: michal.kolodziejski@inea.com.pl

INEA S.A.

Wysogotowo, Wierzbowa st 84

62-081 Przeźmierowo

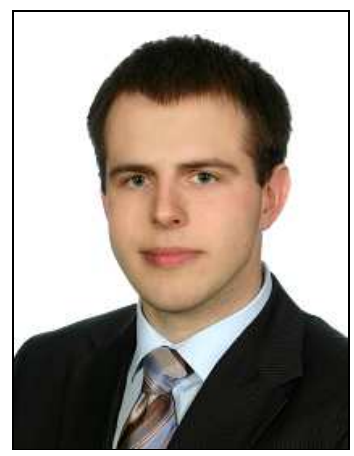

Paweł Kryszkiewicz received his M.Sc. degree in Telecommunications from Poznan University of Technology in 2010. Since 2010 he has been employed at the Chair of Wireless Communications in the Faculty of Electronics and Telecommunications of Poznan University of Technology, as a senior researcher. His research interests focus mostly on physical layer for the future wireless communications systems. In particular, he is interested in the following: multicarrier modulation schemes, e.g., NCOFDM, their application to cognitive radio systems, problems of spectrum shaping for protection of primary users and mitigation of interference caused by nonlinear effects in radio front-end. Since 2010 he has been a member of IEEE. He was involved in international projects (like NEWCOM\#, COGEU, ACROPOLIS, COST Action IC-0902, COST-Terra).

E-mail: pawel.kryszkiewicz@put.poznan.pl

Chair of Wireless Communications

Faculty of Electronics and Telecommunications

Poznan University of Technology

M. Skłodowskiej-Curie sq. 5

60-965 Poznań, Poland 